\title{
The AST/ALT (De Ritis) Ratio Independently Predicts Adverse Outcomes in Patients with Pyogenic Liver Abscess
}

\section{Huifang Dai}

The second affiliated hospital and Yuying children's hospital of wenzhou medical university Jing Xu ( $\nabla$ xujing_6@126.com)

The second affiliated and Yuying children's hospital of wenzhou medical university https://orcid.org/0000-0002-7543-8774

\section{Research article}

Keywords: pyogenic liver abscess, aspartate aminotransaminase, alanine aminotransaminase, poor prognosis, mortality

Posted Date: May 6th, 2020

DOl: https://doi.org/10.21203/rs.3.rs-25277/v1

License: (a) (i) This work is licensed under a Creative Commons Attribution 4.0 International License. Read Full License 


\section{Abstract}

Background: An elevated aspartate aminotransaminase to alanine aminotransaminase ratio (AST/ALT) displays relationships to poor prognosis in viral hepatitis. However, the association between AST/ALT and pyogenic liver abscess (PLA) is unclear.

Aim:This study delved into the relationships of AST/ALT and unfavorable results of PLA cases and ascertained AST/ALT predicting significance.

Methods: In total, 240 PLA cases were consecutively recruited here and underwent a 3-month followingup. This study carried out receiver-operating characteristic (ROC) curve study, multiple-variate logistic regression analyses and univariate analysis.

Results: In line with the receiver-operating curve of AST/ALT for unfavorable results, the cases fell to two subgroups with the use of a threshold of 0.97. Cases with high AST/ALT had higher risk of mortality (16.5\% vs $2.9 \%)$, metastatic infection ( $19.1 \%$ vs $9.7 \%)$, acute hepatic failure ( $7.3 \%$ vs $2.2 \%)$, acute myocardial ( $7.3 \%$ vs $0.7 \%)$, empyema ( $23.7 \%$ vs $10.4 \%$ ) and septic shock ( $13.7 \%$ vs $4.4 \%)$ than cases with low AST/ALT (all P< 0.05). after adjusting for potential confounders in our logistic model, high AST/ALT was independently associated with all unfavorable results $(\mathrm{OR}=4.03,95 \% \mathrm{Cl}=2.12-7.66)$ and death (odds ratio $(\mathrm{OR})=6.17,95 \%$ of confidence interval $(\mathrm{Cl})=1.88-20.26)$. AST/ALT exhibited the widest area underneath ROC curve (AUC) as compared with AST, ALT in death prediction (AUC $=0.821$, cutoff value $=$ $1.31, P<0.01)$ and overall unfavorable results $(A U C=0.690$, cutoff value $=0.97, P<0.01)$.

Conclusion: AST/ALT may be a good candidate for predicting prognosis in future clinical practice of PLA.

\section{Introduction}

Though being a not common disease, pyogenic liver abscess (PLA) seriously threaten individual life, achieving the incidence from 2.3 cases per 10,000 patients living in hospital in North America to 275.4 cases per 100,100 patients living in hospital in Taiwan[1, 2]. For optimized diagnosis-related modality and suitable treating process, PLA mortality is noticeably down-regulated from 10-40\%[3, 4]. Nevertheless, diagnostic and therapeutic problems remain[5]. In emergency department, prognostic markers should be identified for offering more well-timed and aggressing resuscitating process to cases, and plan treating process of cases with PLA subsequently to achieve effective prognostic process.

The aminotransaminases, covering alanine aminotransaminase (ALT) and aspartate aminotransaminase (AST), refer to enzymes produced by liver cells and flowing in the blood stream, denoting hepatocyte impairment; for this reason, the mentioned are considered partial panel required frequently, ascertaining the liver functions[6]. De Ritis first presented the ratio of AST's and ALT's serum activities in 1957, termed as the De Ritis ratio (AST/ALT)[7]. Though it is first proposed as a feature of viral hepatitis, such proportion appears as a conducive biomarker for other diseases[8]. A number of studies recently show that aminotransaminases in a range of levels, covering the De Ritis ratio, can act as effective prognosis- 
associated biomarkers in cases carrying malignant tumor $[9,10]$. Inflammation-associated components, covering serum AST and ALT, display correlations to oxidative stress and systemic inflammation [11]. Oxidative stress in turn, is related to prognosis.

Studies highlighting relationships between AST/ALT and the prognosis of PLA cases are limited. This work aimed at investigating the association of AST/ALT with PLA and the prognostic value of AST/ALT in identifying unfavorable results in PLA cases.

\section{Methods}

\section{Study design}

The medicine-related data of overall PLA cases receiving the admission to the Second Affiliated Hospital and Yuying Children's Hospital of Wenzhou Medical University from Jan 2014 to Dec 2016 were retrospectively reviewed. According to the International Classification of Diseases (Revision 9) search agency database, this study achieved the retrieve of 240 hospitalized PLA cases. The Ethics Committee of the Second Affiliated Hospital of Wenzhou Medical University (No. LCKY2017-01) approved this work, and all subjects provided the informed consent in line with the Declaration of Helsinki in a written form.

\section{Study population}

Included standards: Cases with at least one lesion in the image of liver, covering ultrasound (US) or computed tomography (CT), or in which the whole radiological abnormalities regression is achieved or the positive blood pus culture is achieved based on the antimicrobial medical treatment. Excluded standards: Cases carrying amoebic liver abscesses and cirrhosis.

\section{Data collection and outcome measurements}

The clinical records of cases were retrospectively reviewed, highlighting cases' demographic features, covering the gender and age; comorbid diseases (e.g., hypertension and diabetes mellitus); clinics-related characteristics, covering systolic and diastolic blood pressure (BP), height and temperature as well as body weight; Laboratory findings covering klebsiella pneumoniae (KP) infection, total bilirubin, aspartate transaminase (AST), alanine aminotransferase (ALT), uric acid, albumin, serum creatinine, low-density lipoprotein cholesterol (LDL-C), triglyceride (TG), total cholesterol (TC), high-density lipoprotein cholesterol (HDL-c), activated partial thromboplastin time (APTT), platelet (PLT), prothrombin time (PT), procalcitonin, C-reactive protein (CRP), platelet (PLT) count, hemoglobin $(\mathrm{Hb})$, red blood cell (RBC), neutrophil (N) count, white blood cell (WBC) count. The mentioned results originated from separate medicine-related recordings on admission in line with the definitions specified in advance. 
Hypertension refers to, a systolic blood pressure of $\geq 140 \mathrm{mmHg}$, and/or a diastolic blood pressure of $\geq$ $90 \mathrm{mmHg}$, the recent utilization of antihypertensive drugs. Diabetes mellitus refers to a casual glucose data of $\geq 11.1 \mathrm{mmol} / \mathrm{L}$, a fasting glucose data of $\geq 7.0 \mathrm{mmol} / \mathrm{L}$, the recent use of antidiabetic drugs. Anemia refers to hemoglobin $<13 \mathrm{~g} / \mathrm{dl}$ in men and $<12 \mathrm{~g} / \mathrm{dl}$ in women. This study obtained body mass index $(\mathrm{BMI})$ as weight $(\mathrm{Kg})$ divided by height squared $\left(\mathrm{m}^{2}\right)$.

This study took the record of the treating process of respective case in line with microbiology detecting process and image outcomes, covering antibiotics separately, antibiotics + percutaneous catheter drainage (PCD), or antibiotics + surgery and unfavorable results, e.g., upper gastrointestinal (UGI) bleeding (namely, hemorrhage in association with pressure based on endoscopic observing process, with bright red blood); acute renal failure in hospitalization (the serum creatinine up-regulated by $0.5 \mathrm{mg} / \mathrm{dL}$ from baseline); hospitalized acute myocardial infarction (part of acute myocardial infarction); acute hepatic failure (namely, the growth of serious acute liver injury accompanied by coagulopathy and encephalopathy); acute respiratory failure (namely, cases' requirement for the mechanicsrelatedventilating process); septic shock (the definition and treating process comply with the Surviving Sepsis Campaign criteria)[12]; as well as empyema, mortality, metastatic infection (namely, distal infection of which the bacterium as PLA culture are idential). Moreover, the mortality of cases for three months was identified here.

\section{Statistical analysis}

This study showed continuing information as median [interquartile range] or means \pm standard deviations while making an independent t experiment or a Mann-Whitney $U$ experiment. They also showed categoryrelated data as frequencies (\%) and implemented Fisher's exact or chi-squared experiment. Pearson relation was adopted for estimating the correlations of hemoglobin, PT, BMI and AST/ALT albumin. By univariate logistic regression analysis, the likely predicting elements were ascertained; the mentioned predicting elements were reported causing all unfavorable results or death. This study took respective parameter, and $\mathrm{P}$ value below 0.05 was achieved in accordance with the first univariate study outcomes. A forward stepwise variable taking procedure was conducive to enetually determining the albumin, PT, PLT, hypertension, AST/ALT used in the minimum mode of multiple-variable logistic regression study. For classifying all unfavorable results and the death of these PLA cases subsequently, this study drew a receiver operating characteristic (ROC) curve. The IBM SPSS Statistics 20.0, statistical computer programs (SPSS Inc., USA), better study the mentioned information; P below 0.05 was noticeable.

\section{Results}

\section{Baseline features}

This study recruited 240 cases under PLA totally, the average age reached $67.6 \pm 10.7$ years, $60.8 \%$ belonged to male, and the average AST/ALT reached 1.05 \pm 0.61 . Table 1 lists the fundamental features of survivor and non-survivors. As compared with survivor cases, non-survivor cases were older age, 
exhibiting lower HDL-C, TC, albumin, hemoglobin and RBC, while larger AST/ALT, procalcitonin, PT extents, and detected ratio of non-klebsiella pneumoniae. In line with the maximum Youden index value, the cutoff value for the AST/ALT was set at 0.97; therefore, cases with an AST/ALT $\geq 0.97$ were included in the high AST/ALT cohort, and the cases exhibiting an AST/ALT ratio $<0.97$ were covered in the low AST/ALT cohort, as shown in Table 2. In contrast to low AST/ALT cases, high AST/ALT cases were older age, with significantly higher procalcitonin, APTT and PT levels. Cohort with high AST/ALT exhibited noticeably lower RBC and hemoglobin levels in contrast to cohort with low AST/ALT. 
Table 1

the comparison of the clinical and liver imaging between survivors and non-survivors groups.

\begin{tabular}{|c|c|c|c|c|}
\hline & $\begin{array}{l}\text { Overall } \\
(n=240)\end{array}$ & survivors ( $n=219$ ) & Non-survivors $(n=21)$ & $\mathbf{P}$ \\
\hline Age, years & $67.6 \pm 10.7$ & $66.8 \pm 13.6$ & $76.6 \pm 11.2$ & 0.002 \\
\hline Male & $146(60.8)$ & $135(61.6)$ & $11(52.4)$ & 0.635 \\
\hline $\mathrm{BMI}, \mathrm{Kg} / \mathrm{m}^{2}$ & $23.3 \pm 2.9$ & $23.4 \pm 2.9$ & $22.0 \pm 2.8$ & 0.099 \\
\hline SBP, mmHg & $125 \pm 20$ & $125 \pm 21$ & $130 \pm 16$ & 0.257 \\
\hline $\mathrm{DBP}, \mathrm{mmHg}$ & $71 \pm 11$ & $71 \pm 11$ & $73 \pm 11$ & 0.442 \\
\hline Temperature, ${ }^{\circ} \mathrm{C}$ & $39.3 \pm 0.8$ & $39.4 \pm 0.8$ & $39.1 \pm 0.9$ & 0.360 \\
\hline White blood cell, $10^{9} / \mathrm{L}$ & $12.2 \pm 6.0$ & $12.1 \pm 5.9$ & $13.3 \pm 7.1$ & 0.351 \\
\hline Neutrophil count, $10^{9} / \mathrm{L}$ & $10.17 \pm 5.89$ & $10.06 \pm 5.85$ & $11.41 \pm 6.39$ & 0.146 \\
\hline Temperature, ${ }^{\circ} \mathrm{C}$ & $39.3 \pm 0.8$ & $39.4 \pm 0.8$ & $39.1 \pm 0.9$ & 0.360 \\
\hline Red blood cell, $10^{12} / \mathrm{L}$ & $4.0 \pm 0.6$ & $4.0 \pm 0.6$ & $3.6 \pm 0.5$ & 0.006 \\
\hline Hemoglobin, g/L & $118.9 \pm 18.3$ & $120.1 \pm 18.0$ & $105.9 \pm 17.0$ & 0.001 \\
\hline Platelet count, $10^{9} / \mathrm{L}$ & $222.9 \pm 133.9$ & $226.1 \pm 127.2$ & $188.5 \pm 193.8$ & 0.309 \\
\hline C-reaction protein, $\mathrm{mg} / \mathrm{L}$ & $90.3 \pm 68.8$ & $91.3 \pm 70.7$ & $76.4 \pm 33.5$ & 0.584 \\
\hline Procalcitonin, $\mathrm{pg} / \mathrm{ml}$ & $13.7 \pm 23.6$ & $12.1 \pm 22.1$ & $27.4 \pm 31.9$ & 0.044 \\
\hline Creatinine, umol/L & $72.7 \pm 54.1$ & $71.9 \pm 55.1$ & $81.9 \pm 40.9$ & 0.489 \\
\hline Total bilirubin, $\mathrm{mg} / \mathrm{L}$ & $16.2 \pm 14.5$ & $16.0 \pm 14.3$ & $18.6 \pm 16.8$ & 0.535 \\
\hline$A L T, U / L$ & $71 \pm 72$ & $73 \pm 73$ & $49 \pm 53$ & 0.145 \\
\hline AST, U/L & $68 \pm 86$ & $66 \pm 79$ & $95 \pm 139$ & 0.134 \\
\hline AST/ALT & $1.05 \pm 0.61$ & $0.98 \pm 0.51$ & $1.85 \pm 0.88$ & $<0.001$ \\
\hline Albumin, g/L & $30.8 \pm 6.7$ & $31.3 \pm 6.7$ & $25.3 \pm 4.6$ & $<0.001$ \\
\hline Uric acid, umol/L & $241 \pm 240$ & $239 \pm 91$ & $265 \pm 134$ & 0.291 \\
\hline $\mathrm{TC}, \mathrm{mmol} / \mathrm{L}$ & $3.5 \pm 1.0$ & $3.6 \pm 1.0$ & $3.1 \pm 0.8$ & 0.049 \\
\hline
\end{tabular}

Values are expressed as mean \pm SD or number (\%); ALT, alanine aminotransferase; AST, aspartate Transaminase; TC, total cholesterol; TG, triglyceride; HDL-C, high-density lipoprotein cholesterol; LDL-C, low-density lipoprotein cholesterol; KP, klebsiella pneumoniae; PT, prothrombin time; APTT, activated partial thromboplastin time 


\begin{tabular}{|c|c|c|c|c|}
\hline & $\begin{array}{l}\text { Overall } \\
(n=240)\end{array}$ & survivors $(n=219)$ & Non-survivors $(n=21)$ & $\mathbf{P}$ \\
\hline $\mathrm{TG}, \mathrm{mmol} / \mathrm{L}$ & $1.5 \pm 0.9$ & $1.5 \pm 0.9$ & $1.5 \pm 0.8$ & 0.793 \\
\hline $\mathrm{HDL}-\mathrm{C}, \mathrm{mmol} / \mathrm{L}$ & $0.6 \pm 0.4$ & $0.7 \pm 0.4$ & $0.4 \pm 0.3$ & 0.011 \\
\hline LDL-C, mmol/L & $2.1 \pm 0.8$ & $2.1 \pm 0.8$ & $1.7 \pm 0.7$ & 0.074 \\
\hline PT, s & $14.9 \pm 1.6$ & $14.8 \pm 1.6$ & $15.8 \pm 1.5$ & 0.007 \\
\hline APTT, s & $41.8 \pm 6.0$ & $41.8 \pm 5.8$ & $41.2 \pm 7.2$ & 0.615 \\
\hline KP infection & $180(74.8)$ & $169(77.7)$ & $11(53.8)$ & 0.047 \\
\hline \multicolumn{5}{|l|}{ Abscess features } \\
\hline Solitary lesion & $189(78.8)$ & $175(80.3)$ & $14(65.0)$ & 0.147 \\
\hline Mean size of abscess, $\mathrm{cm}$ & $6.3 \pm 2.8$ & $6.3 \pm 2.8$ & $6.0 \pm 2.9$ & 0.617 \\
\hline \multicolumn{5}{|c|}{$\begin{array}{l}\text { Values are expressed as mean } \pm \text { SD or number (\%); } \mathrm{ALT} \text {, alanine aminotransferase; } \mathrm{AST} \text {, aspartate } \\
\text { Transaminase; TC, total cholesterol; TG, triglyceride; HDL-c, high-density lipoprotein cholesterol; } \mathrm{LDL}-\mathrm{C} \text {, } \\
\text { low-density lipoprotein cholesterol; KP, klebsiella pneumoniae; PT, prothrombin time; APTT, activated } \\
\text { partial thromboplastin time }\end{array}$} \\
\hline
\end{tabular}


Table 2

the comparison of the clinical and liver imaging between low AST/ALT and high AST/ALT groups.

\begin{tabular}{|c|c|c|c|c|}
\hline & $\begin{array}{l}\text { Overall } \\
(n=240)\end{array}$ & $\begin{array}{l}\text { Low AST/ALT }(n= \\
137)\end{array}$ & $\begin{array}{l}\text { High AST/ALT }(n= \\
\text { 103) }\end{array}$ & $\mathbf{P}$ \\
\hline Age, years & $67.6 \pm 10.7$ & $64.7 \pm 13.7$ & $71.3 \pm 12.7$ & $\begin{array}{l}< \\
0.001\end{array}$ \\
\hline Male & $146(60.8)$ & $90(65.7)$ & $56(54.4)$ & 0.083 \\
\hline $\mathrm{BMI}, \mathrm{Kg} / \mathrm{m}^{2}$ & $23.3 \pm 2.9$ & $23.6 \pm 2.8$ & $22.9 \pm 3.0$ & 0.091 \\
\hline SBP, mmHg & $125 \pm 20$ & $125 \pm 21$ & $125 \pm 20$ & 0.826 \\
\hline DBP, mmHg & $71 \pm 11$ & $71 \pm 11$ & $71 \pm 11$ & 0.934 \\
\hline Temperature, ${ }^{\circ} \mathrm{C}$ & $39.3 \pm 0.8$ & $39.4 \pm 0.8$ & $39.3 \pm 0.7$ & 0.416 \\
\hline White blood cell, $10^{9} / \mathrm{L}$ & $12.2 \pm 6.0$ & $12.4 \pm 6.2$ & $11.9 \pm 5.8$ & 0.508 \\
\hline Neutrophil count, $10^{9} / \mathrm{L}$ & $10.17 \pm 5.89$ & $10.34 \pm 6.13$ & $9.94 \pm 5.59$ & 0.607 \\
\hline Red blood cell, $10^{12} / \mathrm{L}$ & $4.0 \pm 0.6$ & $4.1 \pm 0.6$ & $3.9 \pm 0.6$ & 0.003 \\
\hline Hemoglobin, g/L & $118.9 \pm 18.3$ & $121.5 \pm 17.6$ & $115.5 \pm 18.7$ & 0.013 \\
\hline Platelet count, $10^{9} / \mathrm{L}$ & $222.9 \pm 133.9$ & $219.7 \pm 115.7$ & $227.2 \pm 155.2$ & 0.669 \\
\hline C-reaction protein, $\mathrm{mg} / \mathrm{L}$ & $90.3 \pm 68.8$ & $97.2 \pm 77.8$ & $78.1 \pm 47.7$ & 0.179 \\
\hline Procalcitonin, pg/ml & $13.7 \pm 23.6$ & $10.3 \pm 18.0$ & $17.9 \pm 28.7$ & 0.047 \\
\hline Creatinnine, umol/L & $72.7 \pm 54.1$ & $72.1 \pm 63.0$ & $73.5 \pm 39.5$ & 0.839 \\
\hline Total bilirubin, mg/L & $16.2 \pm 14.5$ & $15.0 \pm 10.9$ & $17.7 \pm 18.1$ & 0.146 \\
\hline Albumin, g/L & $30.8 \pm 6.7$ & $31.4 \pm 6.4$ & $30.1 \pm 7.1$ & 0.136 \\
\hline Uric acid, umol/L & $241 \pm 95$ & $238 \pm 88$ & $246 \pm 103$ & 0.513 \\
\hline $\mathrm{PT}, \mathrm{s}$ & $14.9 \pm 1.6$ & $14.7 \pm 1.4$ & $15.1 \pm 1.8$ & 0.029 \\
\hline APTT, s & $41.8 \pm 6.0$ & $41.0 \pm 5.7$ & $42.9 \pm 6.2$ & 0.014 \\
\hline KP infection & $180(74.8)$ & 112 (82.1) & 68 (66.7) & 0.078 \\
\hline \multicolumn{5}{|l|}{ Abscess features } \\
\hline Solitary lesion & 189 (78.8) & 108 (78.8) & $81(78.8)$ & 1.000 \\
\hline
\end{tabular}

Values are expressed as mean \pm SD or number (\%); ALT, alanine aminotransferase; AST, aspartate Transaminase; KP, klebsiella pneumoniae; PT, prothrombin time; APTT, activated partial thromboplastin time 


\begin{tabular}{|c|c|c|c|c|}
\hline & $\begin{array}{l}\text { Overall } \\
(n=240)\end{array}$ & $\begin{array}{l}\text { Low AST/ALT }(n= \\
137)\end{array}$ & $\begin{array}{l}\text { High AST/ALT }(n= \\
\text { 103) }\end{array}$ & $\mathbf{P}$ \\
\hline $\begin{array}{l}\text { Mean size of abscess, } \\
\mathrm{cm}\end{array}$ & $6.3 \pm 2.8$ & $6.2 \pm 2.7$ & $6.4 \pm 2.9$ & 0.637 \\
\hline \multicolumn{5}{|c|}{$\begin{array}{l}\text { Values are expressed as mean } \pm \text { SD or number }(\%) ; A L T \text {, alanine aminotransferase; } A S T \text {, aspartate } \\
\text { Transaminase; KP, klebsiella pneumoniae; } \mathrm{PT} \text {, prothrombin time; APTT, activated partial } \\
\text { thromboplastin time }\end{array}$} \\
\hline
\end{tabular}

\section{Pearson correlation}

Pearson correlation between AST/ALT, PT, BMl, hemoglobin and albumin is shown in Fig. 1. AST/ALT displayed negative relationships to $B M I(r=-0.148, P=0.022)$, albumin $(r=-0.191, P=0.003)$, hemoglobin $(r=-0.249, P<0.001)$ and was related to PT in a positive manner $(r=0.275, P<0.001)$.

\section{Clinical outcomes}

The high AST/ALT cohort showed noticeably larger hospitalizing expense $(P<0.001)$ in contrast to low AST/ALT group. The incidence $(P=0.001)$ of all unfavorable results of cases in the cohort exhibiting small AST/ALT was up-regulated by $27.5-47.4 \%$ from the $19.9 \%$ in the cohort of high AST/ALT, covering the acute hepatic failure (7.3\% vs $2.2 \%)$, metastatic infection ( $19.1 \%$ vs $9.7 \%)$, larger mortality $(16.5 \% \%$ vs $2.9 \%)$, acute myocardial ( $7.3 \%$ vs $0.7 \%)$, empyema ( $23.7 \%$ vs $10.4 \%$ ) and septic shock ( $13.7 \%$ vs $4.4 \%)$ (Table 3). 
Table 3

the comparison of the treatment and clinical outcome between low AST/ALT and high AST/ALT groups.

\begin{tabular}{|c|c|c|c|c|}
\hline & $\begin{array}{l}\text { Overall }(n= \\
240)\end{array}$ & $\begin{array}{l}\text { Low } \\
\text { AST/ALT } \\
(n=137)\end{array}$ & $\begin{array}{l}\text { High AST/ALT }(n= \\
\text { 103) }\end{array}$ & $\mathbf{P}$ \\
\hline Percutaneous drainage & $126(52.5)$ & $71(51.8)$ & $55(53.4)$ & 0.896 \\
\hline Operation & $4(1.7)$ & $2(1.5)$ & $2(1.9)$ & 1.000 \\
\hline Hospital length of stay, days & $17.6 \pm 9.2$ & $17.0 \pm 8.2$ & $18.3 \pm 10.3$ & 0.272 \\
\hline $\begin{array}{l}\text { Hospitalization expenses, } * 10^{4} \\
\text { CNY }\end{array}$ & $3.2 \pm 2.5$ & $2.7 \pm 1.4$ & $4.0 \pm 3.3$ & $\hat{0.001}$ \\
\hline Adverse outcomes & $75(31.3)$ & $27(19.9)$ & $48(47.4)$ & $\hat{0.001}$ \\
\hline Mortality & $21(8.8)$ & $4(2.9)$ & $17(16.5)$ & $\hat{0}_{0.001}$ \\
\hline Metastatic infection & $33(13.6)$ & $13(9.7)$ & $20(19.1)$ & 0.005 \\
\hline Acute renal failure & $9(3.9)$ & $3(2.2)$ & $6(6.2)$ & 0.169 \\
\hline Acute hepatic failure & $10(4.3)$ & $3(2.2)$ & $7(7.3)$ & 0.048 \\
\hline Acute respiratory failure & $11(4.7)$ & $5(3.7)$ & $6(6.2)$ & 0.533 \\
\hline Acute myocardial infarction & $8(3.4)$ & $1(0.7)$ & $7(7.3)$ & 0.010 \\
\hline UGI bleeding & $18(7.8)$ & $7(5.2)$ & $11(11.3)$ & 0.134 \\
\hline empyema & $38(15.9)$ & $14(10.4)$ & $24(23.7)$ & 0.010 \\
\hline Septic shock & $20(8.2)$ & $6(4.4)$ & 14 (13.7) & 0.015 \\
\hline
\end{tabular}

\section{Univariate and multiple-variate logistic regression analysis}

Univariate study verified the associations among age $\geq 65, \mathrm{BMI}<24$, albumin $<30$, anemia, $\mathrm{PLT}<125$, PT $>14.8 \mathrm{~s}, \mathrm{AST} / \mathrm{ALT}>0.97$ and death; nevertheless, age $\geq 65$, albumin $<30$, anemia, $\mathrm{PLT}<125$, diabetes, hypertension, AST/ALT >0.97 displayed relationships to the unfavorable results. With the use of multiplevariate analysis albumin < 30, PT > $14.8 \mathrm{~s}$ and AST/ALT >0/97 were significant predictors for the death; albumin $<30, \mathrm{PLT}<125$, hypertension and AST /ALT $>0.97$ acted as vital predicting elements in terms of the unfavorable results in PLA cases (Table 4 and Table 5). 
Table 4

Univariate and multivariate logistic regression for risk factors associated with mortality

\begin{tabular}{|c|c|c|}
\hline & Univariate analysis & Multivariate analysis \\
\hline Variable & Odd ratio $(95 \% \mathrm{Cl})$ & Odd ratio $(95 \% \mathrm{Cl})$ \\
\hline age $\geq 65$ years & $4.23(1.48-12.06)$ * & \\
\hline Male & $0.74(0.29-1.87)$ & \\
\hline $\mathrm{BMI}<24$ & $3.52(1.01-12.33)$ & \\
\hline Albumin $<30 \mathrm{~g} / \mathrm{L}$ & $3.74(1.32-10.56)$ & 4.55 (1.33-15.57) \\
\hline Anemia $^{a}$ & $3.56(1.31-9.62)$ * & \\
\hline PLT $<125$ & $3.51(1.38-8.94)$ * & \\
\hline $\mathrm{PT}>14.8 \mathrm{~s}$ & $3.23(1.19-8.72)$ * & $3.90(1.26-12.05)$ \\
\hline Percutaneous drainage & $0.80(0.32-2.02)$ & \\
\hline size $>6 \mathrm{~cm}$ & $1.92(0.64-5.75)$ & \\
\hline creatinine $>1.3 \mathrm{mg} / \mathrm{dL}$ & $2.50(0.65-9.55)$ & \\
\hline Diabetes & $0.71(0.28-1.80)$ & \\
\hline hypertension & $0.84(0.29-2.43)$ & \\
\hline AST/ALT > 0.97 & $6.57(2.14-20.19)$ & $6.17(1.88-20.26)$ \\
\hline \multicolumn{3}{|c|}{${ }^{\text {a}}$ Hemoglobin $<13 \mathrm{~g} / \mathrm{dL}$ in men, $<12 \mathrm{~g} / \mathrm{dL}$ in women; $\mathrm{Cl}$, confidence interval } \\
\hline$\star \mathrm{P}<0.05$ & & \\
\hline
\end{tabular}


Table 5

Univariate and multivariate logistic regression for risk factors associated with adverse outcomes

\begin{tabular}{|c|c|c|}
\hline & Univariate analysis & Multivariate analysis \\
\hline Variable & Odd ratio $(95 \% \mathrm{Cl})$ & Odd ratio $(95 \% \mathrm{Cl})$ \\
\hline age $\geq 65$ years & $2.14(1.22-3.76)^{*}$ & \\
\hline Male & $0.79(0.45-1.39)$ & \\
\hline $\mathrm{BMI}<24$ & $1.72(0.94-3.15)$ & \\
\hline Albumin $<30 \mathrm{~g} / \mathrm{L}$ & $2.45(1.38-4.34)$ & $2.20(1.15-4.23)$ \\
\hline Anemia & $1.81(1.03-3.17)^{*}$ & * \\
\hline PLT $<125$ & $3.11(1.67-5.78)$ * & $2.69(1.34-5.42)$ \\
\hline PT $>14.8 \mathrm{~s}$ & $1.72(0.99-3.01)$ & \\
\hline Percutaneous drainage & $0.71(0.41-1.23)$ & \\
\hline size $>6 \mathrm{~cm}$ & $1.02(0.48-2.15)$ & \\
\hline creatinine $>1.3 \mathrm{mg} / \mathrm{dL}$ & $1.80(0.95-3.39)$ & \\
\hline Diabetes & $1.74(1.00-3.05)$ & \\
\hline hypertension & $1.82(1.00-3.31)$ * & $2.23(1.13-4.43)$ \\
\hline AST/ALT > 0.97 & $3.64(2.04-6.50)$ & $4.03(2.12-7.66)$ \\
\hline \multicolumn{3}{|c|}{${ }^{a}$ Hemoglobin $<13 \mathrm{~g} / \mathrm{dL}$ in men, $<12 \mathrm{~g} / \mathrm{dL}$ in women; $\mathrm{Cl}$, confidence interval } \\
\hline$\star P<0.05$ & & \\
\hline
\end{tabular}

\section{Prognostic value of AST/ALT}

To compare the mortality and all negative prognostic process for the the quality of being predictable among PLA cases, this study drew the ROC curves for AST/ALT, AST, ALT, as shown in Fig. 2a-b. Obviously, AST/ALT achieved the biggest area underneath the ROC curves, and it suggested statisticsrelated implication ( 0.821 for death and 0.690 for all the unfavorable results). The optimum cut-off data of AST/ALT for assessed death reached 1.31; such data of all unfavorable consequence reached 0.97 .

\section{Discussion}

According to the information the authors have acquired, this initially evidenced AST/ALT as a robust predicting marker in terms of unfavorable prognostic process in PLA cases. Such relationships continued 
to exist even after relevant complex elements were regulated, covering age, sex, BMI, albumin, PLT, PT, diabetes and hypertension. According to the research, 0.97 is the optimum cutoff for continuing AST/ALT, with AST/ALT > 0.97 at admission displaying relationships to a 4.03 -time larger likelihood of a unfavorable consequence.

De Ritis initially raised AST/ALT idea and the relationships to viral hepatics in 1957[13]. AST/ALT was then employed for estimating the occurrence of progressive fibrosis in chronic hepatic B cases and negative surviving state in chronic viral hepatitis C[14]. AST/ALT recently appears as a prognosis-related biomarker in certain types of malignant tumor and a conducive predicting element in terms of nonhepatic diseases[15-17]. For instance, in a general European cohort of nonmetastatic renal cell carcinoma cases, Bezan and colleagues detected pre-operating AST/ALT as a predicting element of a unfavorable clinics-related consequence[15]. According to Nishikawa and colleagues, preoperative AST/ALT displayed noticeable relationships to a fourfold increase in an unfavorable prognosis in cases with upper urinary tract urothelial carcinoma[18]. Rief and colleagues studied 1782 consecutive cases with peripheral arterial occlusive disease and reported that AST/ALT at admission displayed relationships to critical limb ischemia[19]. Tan and colleagues suggested that AST/ALT is likely to display associations with a risen risk of inferior long period survival in distal cholangiocarcinoma [20]. These existing outcomes verify that AST/ALT increase up-regulates the risk of unfavorable events in a range of diseases, with the thresholds for the mentioned elevations exhibiting similarity to that here.

In terms of baseline feature, we noticed that cases exhibiting high AST/ALT group, who has significantly higher procalcitonin, APTT, PT levels and had less albumin and RBC than those with low AST/ALT cases. Moreover, the AST/ALT is broadly exploited for the assessment of functional liver damage progression and for estimating liver fibrosis degree for more than ten years[21, 22]. In consideration of the several risk indexed displaying relationship to unfavorable results, high AST/ALT represents that physicians are required to adopt rigorous resuscitating process and efficient and suitable treating process.

Existing studies showed that AST/ALT can better detect heavy drinking in the NHANES research than the independent utilization[23]. Our study also noticed that AST/ALT had larger AUCs in contrast to AST and ALT in unfavorable results and mortality estimation ( 0.821 for death and 0.690 for all the unfavorable results). Some existing research showed other prognosis significance in terms of PLA[24-26], covering pleural effusions, concomitant malignancy, multiple abscesses, liver abscess of biliary origin, hemoglobin levels, low albumin, promoted BUN and serum creatinine, and older age. There is no optimal biomarker to estimate liver abscess. Nevertheless, besides other biomarkers, AST/ALT is superior as a biomarker, for its clear, economical efficiency, and easy to get property, and necessitate minimal participation by cases.

The precise system explaining the relationships of poor PLA result with AST/ALT rise has not been clarified, probably for a diversification in activity of ALT and AST. Considerable analyses proved ALT largely increasing in liver tissues and AST showing broad distribution in diverse organs covering the heart, muscle, kidney, and brain, probably making AST continuously more proliferative than ALT even the case exhibits a deteriorating state[14, 15,27]. Among cases achieving a negative consequence, the ALT 
level down-regulation was more obvious than AST's, causing a higher AST/ALT correlated with a unfavorable consequence.

Inflammation-associated parts, covering serum ALT and AST, are more effective predicting elements for liver injury. Besides, ALT is reported displaying relationships to oxidation stress and body inflammations, showing associations with curing reacting processes[28]. The liver is capable of receiving exposure to inner stimuli easily, producing reactive oxygen species (ROS). Inflammation and oxidative stress is likely to get involved in unfavorable PLA results. Nevertheless, broad clinics-related analyses and an growing experiment-related studies should demonstrate the relationships between AST/ALT and PLA prognostic process. Subsequent exploring process of the relevant systems is required.

There are a few limitations in this study. First, an initial retrospective single-center analysis is carried out here, a selecting bias may have occurred. Second, PLA cases' mortality in the hospitalizing course achieved a remarkably low value (21 case of death) and recruited cases were low in amount. As a result, broad multiple-central prospective cohort analyses should demonstrate the outcome. Third, AST and ALT are affected by a range of elements, covering basic case condition and time interval between symptom beginning to hospital visit, not emphasized here.

\section{Conclusions}

In conclusion, AST/ALT was correlated with worse prognoses in PLA cases, and it acted as a separate prognosis-related element all unfavorable results and death. AST/ALT predicting significance to estimate unfavorable results outperforms AST's and ALT's. ALT and AST are the most commonly used serum biomarkers in our daily clinical practice. Subsequent precise longitudinal analyses using bigger samples and wider following-up can better elucidate the effect exerted by AST/ALT as a marker of vital disease or estimating element of poor prognostic process in the PLA cases.

\section{Abbreviations}

PLA, Pyogenic liver abscess; BMI, body mass index; HbA1c, glycosylated hemoglobin; TC, total cholesterol; TG, triglyceride; HDL-c, High density lipoprotein cholesterol; LDL-c, Low density lipoprotein cholesterol

\section{Declarations}

\section{Ethics approval and consent to participate}

This study has obtained the approval from the Ethics Committee of the Second Affiliated Hospital of Wenzhou Medical University (No. LCKY2017-21) and has obtained the written informed consent of all subjects following the Declaration of Helsinki. 


\section{Competing interests}

The authors declare that they have no competing interests.

\section{Availability of data and materials}

The data that support the findings of this study are available from Institutional Review Board of the second affiliated hospital and Yuying Children's Hospital of Wenzhou Medical University but restrictions apply to the availability of these data, which were used under license for the current study, and so are not publicly available. Data are however available from the authors upon reasonable request and with permission of Institutional Review Board of the Second Affiliated Hospital and Yuying Children's Hospital of Wenzhou Medical University.

\section{Funding}

This work was supported by the grants of Project of Zhejiang Provincial Department of Health(2016KYB194), General Research Project of Zhejiang Provincial Education

Department(Y201534290), Wenzhou Science and Technology Bureau(Y20120163). The funders played no role in the design of this study, the collection, analysis and interpretation of data or preparation of the manuscript.

\section{Consent for publication}

Not applicable.

\section{Acknowledgments}

The authors thank the staff at the Department of Endocrinology and Metabolism, the Second Affiliated Hospital and Yuying Children's Hospital of Wenzhou Medical University, and all the patients who participated in the study.

\section{Authors' contributions}

HFD - study concept and design; preparation, review and approval of manuscript. JX - data collection and interpretation; preparation, review and approval of manuscript.

\section{References}


1. Huang CJ, Pitt HA, Lipsett PA, Osterman FA, Jr., Lillemoe KD, Cameron JL, Zuidema GD: Pyogenic hepatic abscess. Changing trends over 42 years. Annals of surgery 1996, 223(5):600-607; discussion 607-609.

2. Law ST, Li KK: Is hepatic neoplasm-related pyogenic liver abscess a distinct clinical entity? World journal of gastroenterology 2012, 18(10):1110-1116.

3. Keller JJ, Tsai MC, Lin CC, Lin YC, Lin HC: Risk of infections subsequent to pyogenic liver abscess: a nationwide population-based study. Clinical microbiology and infection : the official publication of the European Society of Clinical Microbiology and Infectious Diseases 2013, 19(8):717-722.

4. Mischinger HJ, Hauser $H$, Rabl H, Quehenberger F, Werkgartner G, Rubin R, Deu E: Pyogenic liver abscess: studies of therapy and analysis of risk factors. World journal of surgery 1994, 18(6):852857; discussion 858.

5. Alvarez Perez JA, Gonzalez JJ, Baldonedo RF, Sanz L, Carreno G, Junco A, Rodriguez JI, Martinez MD, Jorge Jl: Clinical course, treatment, and multivariate analysis of risk factors for pyogenic liver abscess. American journal of surgery 2001, 181(2):177-186.

6. Ozer J, Ratner M, Shaw M, Bailey W, Schomaker S: The current state of serum biomarkers of hepatotoxicity. Toxicology 2008, 245(3):194-205.

7. Majhi S, Baral N, Lamsal M, Mehta KD: De Ritis ratio as diagnostic marker of alcoholic liver disease. Nepal Medical College journal : NMCJ 2006, 8(1):40-42.

8. Liu Y, Zhao P, Cheng M, Yu L, Cheng Z, Fan L, Chen C: AST to ALT ratio and arterial stiffness in nonfatty liver Japanese population:a secondary analysis based on a cross-sectional study. Lipids in health and disease 2018, 17(1):275.

9. Mori K, Janisch F, Mostafaei H, Kimura S, Lysenko I, Karakiewicz PI, Briganti A, Enikeev DV, Roupret $\mathrm{M}$, Margulis V et al: Prognostic role of preoperative De Ritis ratio in upper tract urothelial carcinoma treated with nephroureterectomy. Urologic oncology 2020.

10. Riedl J, Posch F, Prager G, Eisterer W, Ohler L, Thamer S, Wilthoner K, Petzer A, Pichler P, Hubmann E et al: The AST/ALT (De Ritis) ratio predicts clinical outcome in pancreatic cancer patients treated with first-line nab-paclitaxel and gemcitabine: post-hoc analysis of an Austrian multicenter, noninterventional study. Annals of oncology : official journal of the European Society for Medical Oncology 2019, 30 Suppl 4:iv78.

11. Lin MS, Lin HS, Chung CM, Lin YS, Chen MY, Chen PH, Hu JH, Chou WN, Huang JC, Huang TJ: Serum aminotransferase ratio is independently correlated with hepatosteatosis in patients with HCV: a cross-sectional observational study. BMJ open 2015, 5(9):e008797.

12. Dellinger RP, Levy MM, Rhodes A, Annane D, Gerlach H, Opal SM, Sevransky JE, Sprung CL, Douglas IS, Jaeschke R et al: Surviving Sepsis Campaign: international guidelines for management of severe sepsis and septic shock, 2012. Intensive care medicine 2013, 39(2):165-228.

13. De Ritis F, Coltorti M, Giusti G: An enzymic test for the diagnosis of viral hepatitis; the transaminase serum activities. Clinica chimica acta; international journal of clinical chemistry 1957, 2(1):70-74. 
14. Botros M, Sikaris KA: The de ritis ratio: the test of time. The Clinical biochemist Reviews 2013, 34(3):117-130.

15. Bezan A, Mrsic E, Krieger D, Stojakovic T, Pummer K, Zigeuner R, Hutterer GC, Pichler M: The Preoperative AST/ALT (De Ritis) Ratio Represents a Poor Prognostic Factor in a Cohort of Patients with Nonmetastatic Renal Cell Carcinoma. The Journal of urology 2015, 194(1):30-35.

16. Hu X, Yang WX, Wang Y, Shao YX, Xiong SC, Li X: The prognostic value of De Ritis (AST/ALT) ratio in patients after surgery for urothelial carcinoma: a systematic review and meta-analysis. Cancer cell international 2020, 20:39.

17. Wu J, Li S, Wang Y, Hu L: Pretreatment Aspartate Aminotransferase-to-Alanine Aminotransferase (De Ritis) Ratio Predicts the Prognosis of Nonmetastatic Nasopharyngeal Carcinoma. OncoTargets and therapy 2019, 12:10077-10087.

18. Nishikawa M, Miyake H, Fujisawa M: De Ritis (aspartate transaminase/alanine transaminase) ratio as a significant predictor of recurrence-free survival in patients with upper urinary tract urothelial carcinoma following nephroureterectomy. Urologic oncology 2016, 34(9):417 e419-417 e415.

19. Rief P, Pichler M, Raggam R, Hafner F, Gerger A, Eller P, Brodmann M, Gary T: The AST/ALT (De-Ritis) ratio: A novel marker for critical limb ischemia in peripheral arterial occlusive disease patients. Medicine 2016, 95(24):e3843.

20. Tan X, Xiao K, Liu W, Chang S, Zhang T, Tang H: Prognostic factors of distal cholangiocarcinoma after curative surgery: a series of 84 cases. Hepato-gastroenterology 2013, 60(128):1892-1895.

21. Giannini E, Risso D, Botta F, Chiarbonello B, Fasoli A, Malfatti F, Romagnoli P, Testa E, Ceppa P, Testa R: Validity and clinical utility of the aspartate aminotransferase-alanine aminotransferase ratio in assessing disease severity and prognosis in patients with hepatitis $\mathbf{C}$ virus-related chronic liver disease. Archives of internal medicine 2003, 163(2):218-224.

22. Sheth SG, Flamm SL, Gordon FD, Chopra S: AST/ALT ratio predicts cirrhosis in patients with chronic hepatitis C virus infection. The American journal of gastroenterology 1998, 93(1):44-48.

23. Kim SG, Kim HY, Seo JA, Lee KW, Oh JH, Kim NH, Choi KM, Baik SH, Choi DS: Relationship between serum adiponectin concentration, pulse wave velocity and nonalcoholic fatty liver disease. European journal of endocrinology 2005, 152(2):225-231.

24. Chen $\mathrm{CH}$, Wu SS, Chang HC, Chang YJ: Initial presentations and final outcomes of primary pyogenic liver abscess: a cross-sectional study. BMC gastroenterology 2014, 14:133.

25. Xu J, Wang L: Low T3 Syndrome as a Predictor of Poor Prognosis in Patients With Pyogenic Liver Abscess. Frontiers in endocrinology 2019, 10:541.

26. Xu J, Zhou X, Zheng C: The geriatric nutritional risk index independently predicts adverse outcomes in patients with pyogenic liver abscess. BMC geriatrics 2019, 19(1):14.

27. Lee H, Choi YH, Sung HH, Han DH, Jeon HG, Chang Jeong B, Seo SI, Jeon SS, Lee HM, Choi HY: De Ritis Ratio (AST/ALT) as a Significant Prognostic Factor in Patients With Upper Tract Urothelial Cancer Treated With Surgery. Clinical genitourinary cancer 2017, 15(3):e379-e385. 
28. Yamada J, Tomiyama H, Yambe M, Koji Y, Motobe K, Shiina K, Yamamoto Y, Yamashina A: Elevated serum levels of alanine aminotransferase and gamma glutamyltransferase are markers of inflammation and oxidative stress independent of the metabolic syndrome. Atherosclerosis 2006, 189(1):198-205.

\section{Figures}


Figure 1

Scatter diagrams showing correlation between AST/ALT, PT, hemoglobin, BMI and albumin. 
(a)

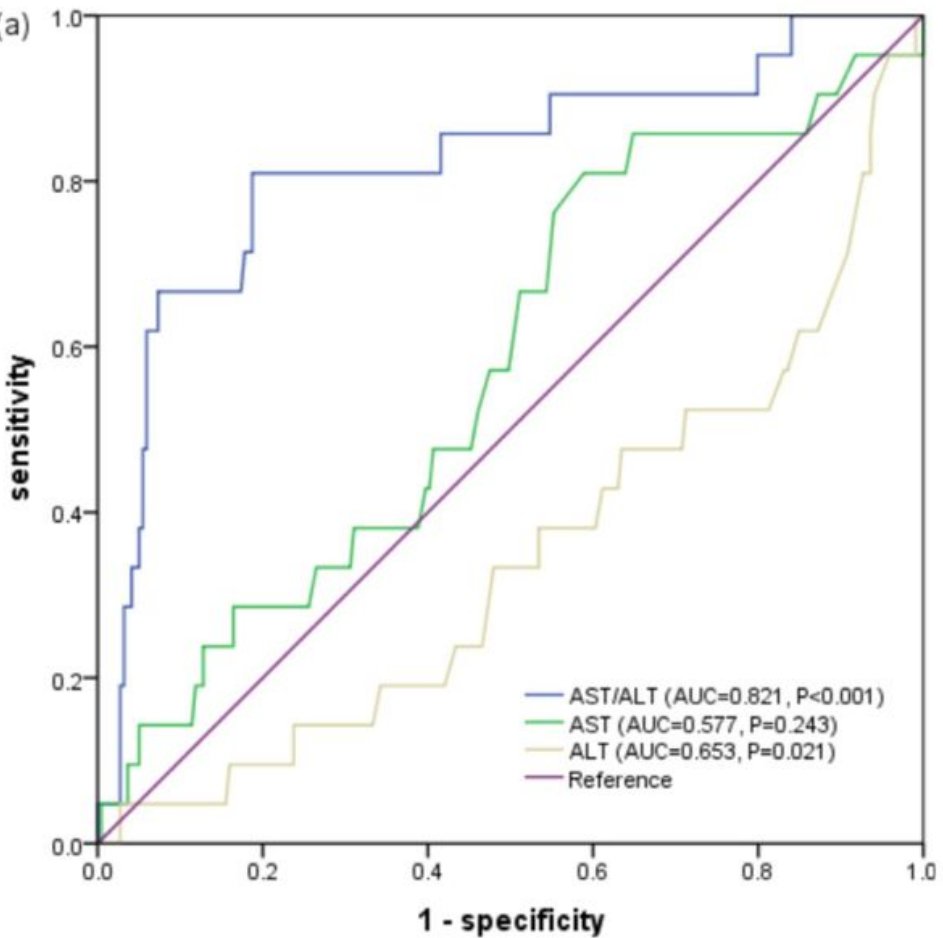

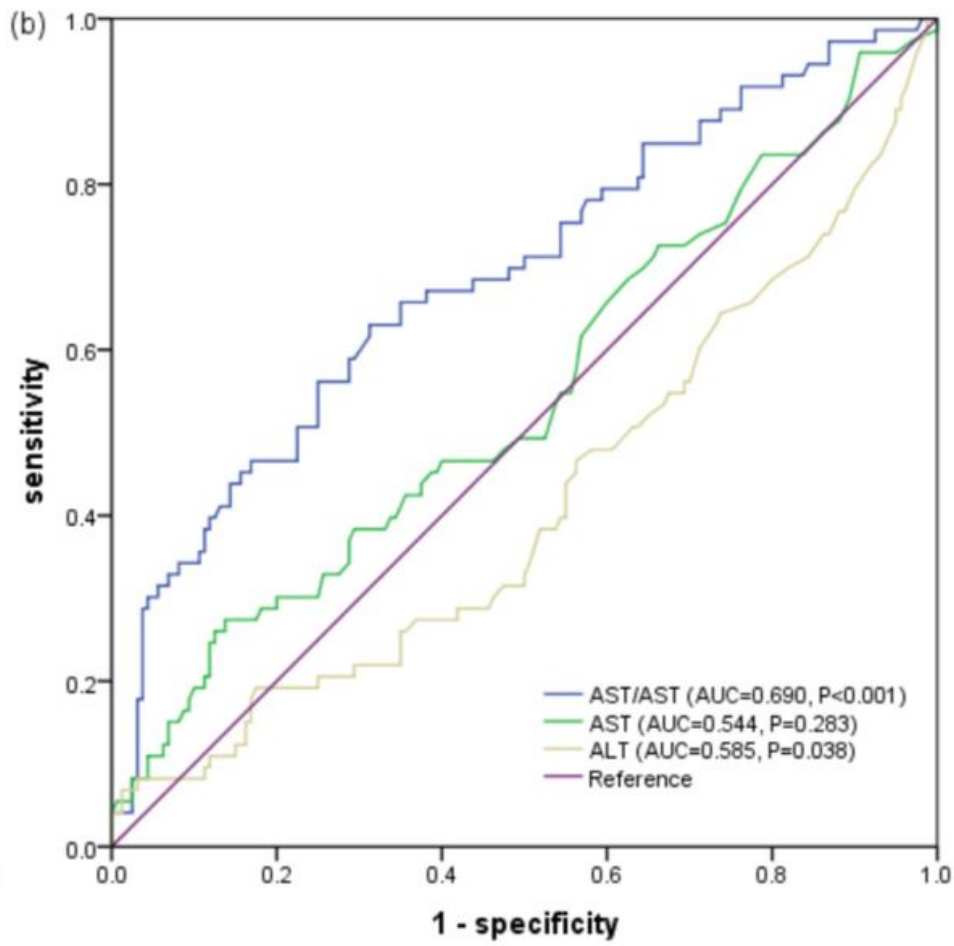

Figure 2

a. ROC analysis of AST/ALT, AST, ALT to death among PLA patients. b. ROC analysis of AST/ALT, AST, ALT to adverse outcomes among PLA patients. 